\begin{tabular}{|c|c|}
\hline Title & Method of determining kinetic boundary conditions in net evaporation/condensation \\
\hline Author(s) & Kon, Misaki; Kobayashi, Kazumichi; W atanabe, Masao \\
\hline Citation & $\begin{array}{l}\text { Physics of Fluids, 26(7), } 072003 \\
\text { https://doi .org/10.1063/1.4890523 }\end{array}$ \\
\hline Issue Date & $2014-07$ \\
\hline Doc URL & http:/hdl.handle.net/2115/56594 \\
\hline Rights & $\begin{array}{l}\text { Copyright } 2014 \text { A merican Institute of Physics. This article may be downloaded for personal use only. A ny other use } \\
\text { requires prior permission of the author and the A merican Institute of Physics. The following article appeared in Physics } \\
\text { of Fluids } 26072003 \text { (2014) and may be found at } \\
\text { http://scitation.aip.org/content/aip/ournal/pof2/26///10.1063/1.4890523. }\end{array}$ \\
\hline Tyре & article \\
\hline File Information & 1.4890523.pdf \\
\hline
\end{tabular}

Instructions for use 


\title{
Method of determining kinetic boundary conditions in net evaporation/condensation
}

\author{
Misaki Kon, Kazumichi Kobayashi, ${ }^{a}$ and Masao Watanabe \\ Division of Mechanical and Space Engineering, Faculty of Engineering, \\ Hokkaido University, Kita 13 Nishi 8, Kita-ku, Sapporo, Hokkaido 060-8628, Japan
}

(Received 31 January 2014; accepted 3 July 2014; published online 23 July 2014)

\begin{abstract}
The aim of the present study is to develop the method of determining the kinetic boundary condition (KBC) at a vapor-liquid interface in net evaporation/condensation. We proposed a novel method for determining the KBC by combining the numerical simulations of the mean field kinetic theory and the molecular gas dynamics. The method was evaluated on steady vapor flow between two liquid slabs at different temperatures. A uniform net mass flux in the vapor phase induced by net evaporation and condensation is obtained from the numerical simulation of the mean field kinetic theory for both vapor and liquid phases. The $\mathrm{KBC}$ was specified by using the uniform net mass flux, and the numerical simulation of the molecular gas dynamics was conducted for the vapor phase. Comparing the macroscopic variables in the vapor phase obtained from both numerical simulations, we can validate the KBC whether the appropriate solutions are obtained. Moreover, the evaporation and condensation coefficients were estimated uniquely. The results showed that the condensation and evaporation coefficients were identical and constant in net evaporation. On the other hand, in net condensation, the condensation coefficient increased with the collision molecular mass flux. We also presented the applicable limit of the KBC which is assumed to be the isotropic Gaussian distribution at the liquid temperature. From these results, the KBCs in net evaporation and condensation, which enable the exact macroscopic variables to be determined, were proposed. @ 2014 AIP Publishing LLC. [http://dx.doi.org/10.1063/1.4890523]
\end{abstract}

\section{INTRODUCTION}

Net evaporation and condensation are the fundamental physical processes underlying vapor flows and heat and mass transfers across a vapor-liquid interface. Since these phenomena originate from the deviation of the velocity distribution function, $f$, composed of the molecules outgoing from or colliding onto the vapor-liquid interface, the application of the kinetic theory of gases (molecular gas dynamics) is essential to examine the vapor behaviors near the interface. ${ }^{1,2}$ In the analysis of the kinetic theory for vapor flows, the kinetic boundary condition (KBC) at the vapor-liquid interface plays an important role in the nonequilibrium region near the interface. ${ }^{1-3}$ If the correct $\mathrm{KBC}$ is established in the various vapor-liquid nonequilibrium states, the exact quantities of mass, momentum, and energy fluxes accompanying net evaporation and condensation can be estimated only from the analysis by the kinetic theory. Since the KBC is adopted for the molecules outgoing from the interface to the vapor phase, the key processes of the molecules at the vapor-liquid interface are evaporation and reflection. For the analysis of the evaporation and condensation phenomena related to the KBC, several studies have been conducted by using molecular dynamics (MD) simulations and other types of molecular simulations. ${ }^{4-24}$ Some of these studies have attempted to distinguish each molecule evaporating from, condensing onto, or reflecting at the vapor-liquid interface

\footnotetext{
a)Electronic mail: kobakazu@eng.hokudai.ac.jp
} 


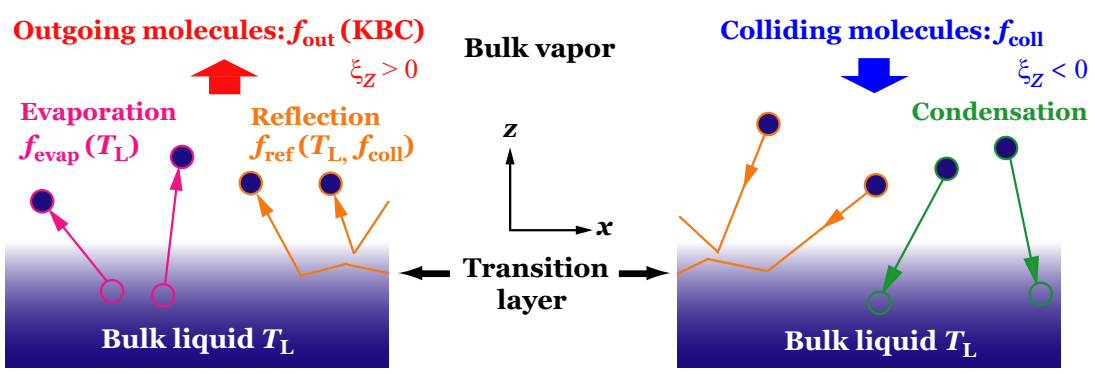

FIG. 1. Schematics of evaporation, reflection and condensation processes of molecules at a vapor-liquid interface according to Eq. (1). Left: the outgoing molecules from the interface, right: the colliding molecules onto the interface.

(e.g., the detailed behaviors of molecules in the vicinity of the vapor-liquid interface are discussed by Cao et al..$^{20}$ ).

In order to avoid the ambiguities introduced in the process of assigning a molecule to evaporation or reflection, Ishiyama et al. ${ }^{4}$ proposed the novel concept of spontaneous evaporation ${ }^{9}$ with the assumption that the evaporation molecular mass flux is only a function of the liquid temperature, and they confirmed the presence of the evaporation mass flux through a virtual vacuum simulation. The general form of the $\mathrm{KBC}$ according to the spontaneous evaporation, which is as shown in Fig. 1, is given as follows: $:^{3,4}$

$$
f_{\text {out }}=f_{\text {evap }}\left(x_{i}, \xi_{i}, T_{\mathrm{L}}\right)+f_{\text {ref }}\left(x_{i}, \xi_{i}, T_{\mathrm{L}}, f_{\text {coll }}\right), \quad \text { for } \xi_{z}>0,
$$

where $f_{\text {evap }}$ and $f_{\text {ref }}$ denote the velocity distribution functions of molecules that spontaneously evaporate from the vapor-liquid interface and that are reflected at the interface, respectively. $x_{i}(x, y$, and $z$ ) denotes the physical coordinates, where $z$ indicates the direction normal to the vapor-liquid interface, and $x$ and $y$ indicate the directions tangential to the interface. $\xi_{i}$ denotes the molecular velocity $\left(\xi_{x}, \xi_{y}\right.$, and $\left.\xi_{z}\right) ; T_{\mathrm{L}}$, the liquid temperature; and $f_{\text {coll }}$, the velocity distribution function of molecules colliding onto the interface. Equation (1) is then rewritten as ${ }^{3}$

$$
f_{\text {out }}=\left[\alpha_{\mathrm{e}} \rho^{*}+\left(1-\alpha_{\mathrm{c}}\right) \sigma\right] \hat{f}_{\text {out }}, \quad \text { for } \xi_{z}>0,
$$

where $\hat{f}_{\text {out }}$ denotes the normalized velocity distribution function. One of the conventional forms of $\hat{f}_{\text {out }}$ is written as follows:

$$
\hat{f}_{\text {out }}^{*}=\frac{1}{\left(\sqrt{2 \pi R T_{\mathrm{L}}}\right)^{3}} \exp \left(-\frac{\xi_{x}^{2}+\xi_{y}^{2}+\xi_{z}^{2}}{2 R T_{\mathrm{L}}}\right), \text { for } \xi_{z}>0,
$$

where $R$ denotes the gas constant. Alternatively, Ishiyama et al..$^{5}$ showed the following $\hat{f}_{\text {out }}$ in net condensation:

$$
\hat{f}_{\text {out }}^{* *}=\frac{1}{\sqrt{2 \pi R T_{\mathrm{n}}}\left(2 \pi R T_{\mathrm{t}}\right)} \exp \left(-\frac{\xi_{z}^{2}}{2 R T_{\mathrm{n}}}-\frac{\xi_{x}^{2}+\xi_{y}^{2}}{2 R T_{\mathrm{t}}}\right), \quad \text { for } \xi_{z}>0,
$$

where $T_{\mathrm{n}}$ and $T_{\mathrm{t}}$ are the normal and tangential temperatures, respectively, composed of the outgoing molecules. Equation (4) has an anisotropic Gaussian distribution affected by $f_{\text {coll }}$ under net condensation. For the distribution of $\hat{f}_{\text {out }}^{* *}\left(T_{\mathrm{L}}, f_{\text {coll }}\right)$ in net condensation, the results obtained in our simulation by using the mean field kinetic theory ${ }^{23}$ were same tendency for those obtained through MD simulations. ${ }^{5}$ As seen from Eqs. (3) and (4), the velocity distribution normal to the interface represents perfect accommodation $\left(T_{\mathrm{n}}=T_{\mathrm{L}}\right)$. On the other hand, in a recent study, ${ }^{6} T_{\mathrm{t}}$ was formulated by using the thermal accommodation coefficient. $\rho^{*}$ in Eq. (2) denotes the saturated vapor density at the liquid temperature $T_{\mathrm{L}}$, and $\sigma$ is related to the collision molecular mass flux $J_{\text {coll }}$ at the interface, which is defined as

$$
\sigma \sqrt{\frac{R T_{\mathrm{L}}}{2 \pi}}=-\int_{\xi_{z}<0} \xi_{z} f_{\text {coll }} \mathrm{d} \xi=J_{\text {coll }}
$$


where $\mathrm{d} \boldsymbol{\xi}=\mathrm{d} \xi_{x} \mathrm{~d} \xi_{y} \mathrm{~d} \xi_{z}, \int_{\xi_{z}<0} \mathrm{~d} \boldsymbol{\xi}=\int_{-\infty}^{0} \mathrm{~d} \xi_{z} \int_{-\infty}^{\infty} \mathrm{d} \xi_{x} \int_{-\infty}^{\infty} \mathrm{d} \xi_{y}$, and $\sigma$ is a parameter having unit of density which is obtained when the velocity distribution function for reflected molecules normal to the interface is the Gaussian distribution at the liquid temperature (Eq. (3) or (4)), and $\alpha_{\mathrm{e}}=\alpha_{\mathrm{c}}=0$ : $\left(1-\alpha_{\mathrm{c}}\right) \sigma \sqrt{R T_{\mathrm{L}} / 2 \pi}$ denotes the mass flux of reflected molecules at the interface when $\alpha_{\mathrm{e}} \neq 0$ and $\alpha_{\mathrm{c}} \neq 0 . \alpha_{\mathrm{e}}$ and $\alpha_{\mathrm{c}}$ denote the evaporation and condensation coefficients, respectively, ${ }^{3,4}$

$$
\alpha_{\mathrm{e}}=\frac{J_{\text {evap }}}{J_{\text {out }}^{*}}, \quad \alpha_{\mathrm{c}}=\frac{J_{\text {cond }}}{J_{\text {coll }}},
$$

where $J_{\text {evap }}$ is the evaporation molecular mass flux, defined as $J_{\text {evap }}=\int_{\xi_{z}>0} \xi_{z} f_{\text {evap }} \mathrm{d} \xi$, where $\int_{\xi_{z}>0} \mathrm{~d} \xi=\int_{0}^{\infty} \mathrm{d} \xi_{z} \int_{-\infty}^{\infty} \mathrm{d} \xi_{x} \int_{-\infty}^{\infty} \mathrm{d} \xi_{y} . J_{\text {out }}^{*}$ is the outgoing molecular mass flux in equilibrium, given as $J_{\text {out }}^{*}=\rho^{*} \sqrt{R T_{\mathrm{L}} / 2 \pi}$. The condensation molecular mass flux is obtained as $J_{\text {cond }}=J_{\text {coll }}-J_{\text {ref }}$, where $J_{\text {ref }}=\int_{\xi_{z}>0} \xi_{z} f_{\text {ref }} \mathrm{d} \xi=\int_{\xi_{z}>0} \xi_{z}\left(f_{\text {out }}-f_{\text {evap }}\right) \mathrm{d} \xi$. By definition, ${ }^{4,9}$ the evaporation coefficient is a function of the liquid temperature. Furthermore, Ishiyama et al. ${ }^{5}$ showed that the values of $\alpha_{\mathrm{e}}$ and $\alpha_{\mathrm{c}}$ were identical and constant in various nonequilibrium states.

Here, according to recent experimental and molecular dynamics studies, several values have been reported for the evaporation and condensation coefficients. From our recent experimental study with a shock tube, ${ }^{25}$ the values of the condensation coefficients of water and methanol decrease with increasing in the magnitude of net condensation. Gu et al. ${ }^{16,17}$ conducted MD simulations to investigate the evaporation and condensation coefficients in vapor-liquid equilibrium and nonequilibrium states without using vacuum evaporation simulations for argon molecules. The evaporation coefficients in vapor-liquid equilibrium states are slightly smaller than those obtained in the vacuum evaporation simulations. ${ }^{16}$ Furthermore, the evaporation and condensation coefficients vary in net condensation. ${ }^{17}$ Cheng et al. ${ }^{21}$ conducted MD simulations for evaporation flows of Lennard-Jones fluids. They showed that the condensation probability increases with the normal translational energy of molecules colliding onto the interface, which is similar to the results of Tsuruta et al. ${ }^{10}$

Thus, ambiguity still exists in terms of the exact values of the evaporation and condensation coefficients in net evaporation and condensation. Moreover, one of the most important issues is that the validations of the KBC, including the determination of the evaporation and condensation coefficients, are still lacking in the literature. Hence, there is no certainty whether the solution by using the obtained $\mathrm{KBC}$ is correct. A method that validates the constructed $\mathrm{KBC}$ for describing the density, velocity, and temperature profiles of vapor in net evaporation and condensation is desired.

In this paper, we propose a novel method for determining the KBC by combining the precise simulations of the Enskog-Vlasov equation based on the mean field kinetic theory and the Boltzmann equation based on the molecular gas dynamics. Since this novel method also validates the KBC, we can construct appropriate KBCs in net evaporation and condensation to describe the exact variables. Our target system is a steady net evaporation and condensation between two liquid slabs at different temperatures, introduced in Sec. II.

\section{METHOD OF DETERMINING KINETIC BOUNDARY CONDITIONS}

Here, we propose the determination method of the $\mathrm{KBCs}$ in net evaporation and condensation in the present study. A schematic of the present simulation configuration is shown in Fig. 2 (this configuration is often called two-surface problem). A space filled with pure vapor is created between two liquid slabs. The liquid temperatures of the left- and the right-hand side slabs are $T_{\mathrm{L} 1}$ and $T_{\mathrm{L} 2}$ $\left(T_{\mathrm{L} 1}>T_{\mathrm{L} 2}\right)$, respectively. Several MD simulations were conducted for this configuration such as Meland $^{26}$ and Frezzotti. ${ }^{12}$ In the molecular gas dynamics, the position where the KBC is prescribed has been conventionally called interface (for example, see Ref. 1). However, at the molecular level, the vapor-liquid interface has the transition layer with a finite thickness. Hence, to avoid the confusion, we refer to the position where the $\mathrm{KBC}$ is prescribed as kinetic boundary as shown in Fig. 2. A uniform and constant net mass flux $\rho v_{z}$ is produced in the vapor as a consequence of steady net evaporation and condensation, where $\rho$ is the vapor density and $v_{z}$ is the vapor velocity in $z$ direction. The flux is evaluated as the first moment of the molecular velocity distribution function, which is numerically calculated based on the mean field kinetic theory by solving the Enskog-Vlasov 

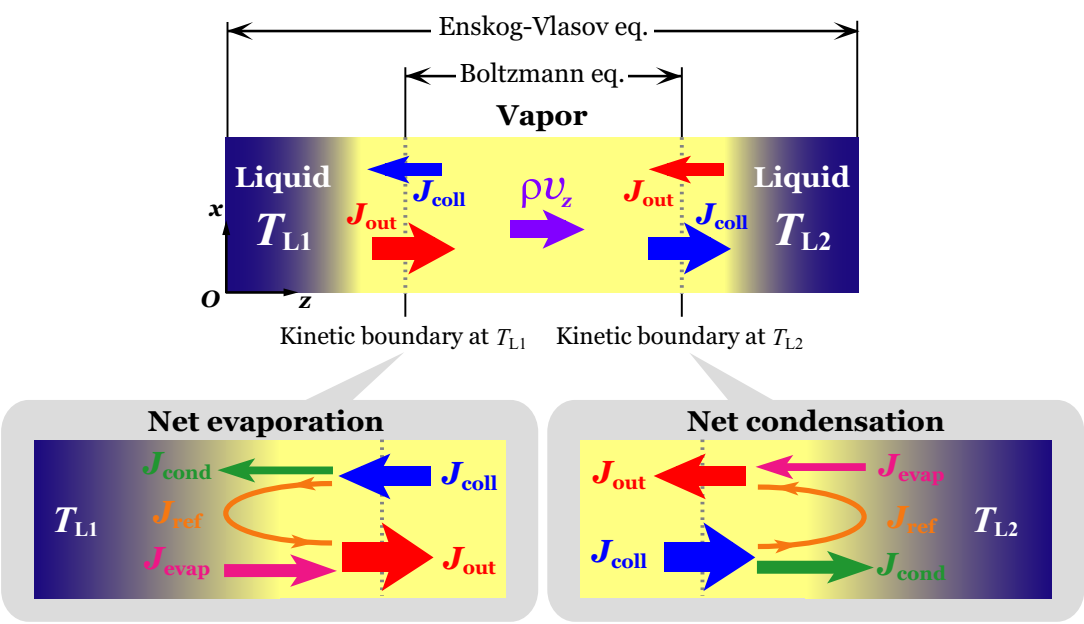

FIG. 2. Schematics of the present simulation configuration. Vapor flow is induced by net evaporation and condensation between two liquid slabs at different temperatures $\left(T_{\mathrm{L} 1}>T_{\mathrm{L} 2}\right)$. The bottom figures show the mass flux relations in net evaporation and condensation.

equation for both of the vapor and liquid phases. In the present study, molecules are regarded as hard spheres with a long-range attractive tail. The great advantage of the use of the Enskog-Vlasov equation is its capability of dealing a large number of particles $\left(1.2 \times 10^{6}\right.$ molecules are adopted in this study), which enable us to conduct the simulations with greater accuracy than those with the MD simulations. ${ }^{12,26}$ Because of this feature, the Enskog-Vlasov equation accurately evaluates even weak net evaporation and condensation induced by small liquid temperature differences $\left(\left(T_{\mathrm{L} 1}\right.\right.$ $\left.-T_{\mathrm{L} 2}\right) / T_{\mathrm{L} 2} \approx O\left(10^{-2}\right)$ ) with small statistical errors. Here, it should be emphasized that the molecular velocity distribution functions for both of the vapor and liquid phases, or for the whole calculation domain, are determined by solving the Enskog-Vlasov equation.

Once the Enskog-Vlasov equation is solved and molecular velocity distribution function is determined, it is all ready to discuss the validity of the KBC (Eq. (2)) for the Boltzmann equation. First, KBCs in net evaporation and condensation are rewritten with the use of the solution of the Enskog-Vlasov equation. The solutions of the Boltzmann equation with the KBCs are then quantitatively compared with those of the Enskog-Vlasov equation. The comparison verifies whether the solutions of the Boltzmann equation can properly reproduce the vapor flow obtained the solutions of the Enskog-Vlasov equation. In the present study, we assume that the functional form of $\hat{f}_{\text {out }}$ in Eq. (2) is $\hat{f}_{\text {out }}^{*}\left(T_{\mathrm{L}}\right)$ as shown in Eq. (3). One aim of this study is to determine the applicable limit of the analysis by using this assumption. We also discuss the values of the evaporation and condensation coefficients determined under this assumption. Recent studies have shown that $f_{\text {out }}$ is affected by the accommodation coefficient of reflected molecules. ${ }^{6,22}$ In this study, we assume the accommodation coefficients normal and tangential to the kinetic boundary are unity. The improvement of the present method by using $f_{\text {out }}$ in consideration of the influence of the accommodation coefficient is the future work.

Let us consider the kinetic boundary at $T_{\mathrm{L} 1}$ in net evaporation. With the use of the evaporation and condensation coefficients, the uniform net mass flux $\rho v_{z}$ in the vapor phase is written as

$$
\rho v_{z}=J_{\text {out }}-J_{\text {coll }}=J_{\text {evap }}+J_{\text {ref }}-\left(J_{\text {cond }}+J_{\text {ref }}\right)=J_{\text {evap }}-J_{\text {cond }}=\left(\alpha_{\mathrm{e}} \rho^{*}-\alpha_{\mathrm{c}} \sigma\right) \sqrt{\frac{R T_{\mathrm{L} 1}}{2 \pi}} .
$$

Substitution of Eq. (7) in Eq. (2) leads to 25,27

$$
f_{\text {out }}=\left[\rho v_{z} \sqrt{\frac{2 \pi}{R T_{\mathrm{L} 1}}}+\sigma\right] \hat{f}_{\text {out }}^{*}\left(T_{\mathrm{L} 1}\right), \quad \text { for } \xi_{z}>0 .
$$


The same procedure can be adopted for the kinetic boundary in net condensation by using the same $\rho v_{z}$ with the mass flux relation as shown in Fig. 2 . The $\mathrm{KBC}$ at the kinetic boundary at $T_{\mathrm{L} 2}$ in net condensation is shown as follows:

$$
f_{\text {out }}=\left[\sigma-\rho v_{z} \sqrt{\frac{2 \pi}{R T_{\mathrm{L} 2}}}\right] \hat{f}_{\text {out }}^{*}\left(T_{\mathrm{L} 2}\right), \quad \text { for } \xi_{z}<0 .
$$

With the use of $\rho v_{z}$ obtained from the solution of the Enskog-Vlasov equation, the appropriate $\mathrm{KBCs}$ are specified at each kinetic boundary as shown in Fig. 2. The unique macroscopic variables, e.g., $\rho, v, T$, etc., of the vapor flow field are obtained by solving the Boltzmann equation with the KBCs given by Eqs. (8) and (9). It should be emphasized that these variables strongly depend on the $\mathrm{KBCs}$; hence, we conclude that the KBCs for the Boltzmann equation are validated if and only if the macroscopic variables obtained from the Boltzmann equation with Eqs. (8) and (9) as the KBCs show a good agreement with those obtained from the Enskog-Vlasov equation.

Moreover, the modification of Eq. (7) leads to an explicit expression for the condensation coefficient if the evaporation coefficient is only the function of the liquid temperature

$$
\alpha_{\mathrm{c}}=-\frac{\rho v_{z}}{\sigma \sqrt{\frac{R T_{L 1}}{2 \pi}}}+\alpha_{\mathrm{e}} \frac{\rho^{*}}{\sigma} .
$$

Equation (10) shows that the condensation coefficient is uniquely determined once the evaporation coefficient is determined for a given value of $T_{\mathrm{L} 1}$ since $\rho^{*}\left(T_{\mathrm{L} 1}\right)$ and $\sigma$ are obtained from the solutions of the Enskog-Vlasov equation and Boltzmann equation, respectively, as shown in Sec. III. The value of the evaporation coefficient can be successfully estimated without the vacuum evaporation simulation ${ }^{4}$ as shown in Sec. IV C.

In two-surface problems, well-known characteristic phenomena occur in certain configurations and have been called as inverted temperature gradient phenomena ${ }^{28}$ and negative mass flows. ${ }^{29}$ Inverted temperature gradient phenomena have been investigated by using MD simulations. ${ }^{12,26,30}$ Moreover, theoretical study was conducted on these phenomena, and the threshold of the occurrence was reported. ${ }^{31}$ Negative mass flows were investigated by Onishi ${ }^{29}$ (but never occurred in the present simulation). Detailed explanations of these phenomena are beyond the scope of this study.

\section{NUMERICAL METHODS}

\section{A. Simulation of the Enskog-Vlasov equation}

The Enskog-Vlasov equation is a practical equation based on the mean field kinetic theory. ${ }^{15,32}$ This equation can describe a hard-sphere fluid under the self-consistent force field generated from the soft attractive tail. In terms of the one-particle velocity distribution function $f$, the Enskog-Vlasov equation is expressed as

$$
\frac{\partial f}{\partial t}+\xi_{i} \frac{\partial f}{\partial x_{i}}+\frac{F_{i}\left(x_{i}, t\right)}{m} \frac{\partial f}{\partial \xi_{i}}=C_{E},
$$

where $m$ is the mass of a hard sphere molecule and $F_{i}$ is the self-consistent force field, determined from the Sutherland potential $\phi$

$$
F_{i}\left(x_{i}, t\right)=\int_{\left\|x_{1 i}-x_{i}\right\|>a} \frac{\mathrm{d} \phi}{\mathrm{d} r} \frac{x_{1 i}-x_{i}}{\left\|x_{1 i}-x_{i}\right\|} n\left(x_{1 i}, t\right) \mathrm{d} x_{1 i}, \quad \phi(r)= \begin{cases}+\infty & (r<a) \\ -\phi_{a}\left(\frac{r}{a}\right)^{-\gamma} & (r \geq a),\end{cases}
$$

where $a$ is the molecular diameter, $r$ is $r=\left\|x_{i 1}-x_{i}\right\|, \phi_{a}$ is a constant parameter, and $\gamma$ is six. The treatment of $F_{i}\left(x_{i}, t\right)$ is similar to that in Frezzotti et al. ${ }^{15}$ The collision term $C_{E}$ is written as

$$
\begin{aligned}
C_{E}= & a^{2} \int\left\{Y\left[n\left(x_{i}+\frac{a}{2} K_{i}, t\right)\right] f\left(x_{i}+a K_{i}, \hat{\xi}_{1 i}, t\right) f\left(x_{i}, \hat{\xi}_{i}, t\right)-Y\left[n\left(x_{i}-\frac{a}{2} K_{i}, t\right)\right] f\left(x_{i}-a K_{i}, \xi_{1 i}, t\right)\right. \\
& \left.\times f\left(x_{i}, \xi_{i}, t\right)\right\} H\left(\xi_{r i} K_{i}\right)\left(\xi_{r i} K_{i}\right) \mathrm{d} \xi_{1} \mathrm{~d}^{2} \boldsymbol{K},
\end{aligned}
$$


where $Y$ is the pair correlation function, $n$ is the number density, $K_{i}$ is the unit vector defined as $K_{i}=\left(x_{1 i}-x_{i}\right) /\left(\left\|x_{1 i}-x_{i}\right\|\right), H$ is the Heaviside function, and $\hat{\xi}_{i}$ and $\hat{\xi}_{1 i}$ are the post-collisional velocity vectors of two colliding molecules. $\xi_{r i}$ is their relative velocity $\xi_{r i}=\xi_{i}-\xi_{1 i}$. The velocity distribution function $f$ is defined as $\mathrm{d} N=(1 / m) f \mathrm{~d} \boldsymbol{x} \mathrm{d} \boldsymbol{\xi}$, where $\mathrm{d} N$ is the number of molecules in an infinitesimal volume element in the 6-dimensional phase space $\mathrm{d} \boldsymbol{x} \mathrm{d} \boldsymbol{\xi}$, where $\mathrm{d} \boldsymbol{x}=\mathrm{d} x \mathrm{~d} y \mathrm{~d} z$. Hence, the macroscopic variables (density, velocity, temperature, etc.) are defined as the moments of $f^{1,2}$

$$
\rho=\int_{-\infty}^{\infty} f \mathrm{~d} \boldsymbol{\xi}, \quad v_{i}=\frac{1}{\rho} \int_{-\infty}^{\infty} \xi_{i} f \mathrm{~d} \boldsymbol{\xi}, \quad T=\frac{1}{3 \rho R} \int_{-\infty}^{\infty}\left(\xi_{i}-v_{i}\right)^{2} f \mathrm{~d} \boldsymbol{\xi}
$$

where $v_{i}$ denotes the velocity $\left(v_{x}, v_{y}\right.$, and $\left.v_{z}\right)$.

In this study, the equation of state is given as follows: ${ }^{33}$

$$
p=\rho R T \frac{1+\eta+\eta^{2}-\eta^{3}}{(1-\eta)^{3}}-\frac{2}{3} \pi a^{3} \frac{\gamma}{\gamma-3} \phi_{a} n^{2}, \quad \eta=\frac{\pi}{6} n a^{3},
$$

where $\eta$ is the atomic packing factor. The critical temperature $T_{\mathrm{C}}$ is obtained from Eq. (15)

$$
T_{\mathrm{C}}=0.094329 \frac{4 \gamma}{\gamma-3} \frac{\phi_{a}}{k},
$$

where $k$ is the Boltzmann constant.

For the Enskog equation, some numerical schemes were proposed based on the direct simulation Monte Carlo (DSMC) method. ${ }^{15,34,35}$ In this study, we utilize the Enskog-DSMC method proposed by Frezzotti. ${ }^{15,34}$ The Enskog-Vlasov equation has previously been investigated for the vapor-liquid system by using the Enskog-DSMC method (for examples, Frezzotti et al. ${ }^{15,36}$ and Kobayashi et al. $\left.{ }^{23}\right)$.

We consider a one-dimensional steady net evaporation and condensation in this study (see Fig. 2). The vapor flow is induced from the left- to the right-hand sides along the $z$ axis. The origin of the $z$ axis is at the left edge in Fig. 2. Figure 3 shows the actual system of the present simulation. The length of the system $L_{\mathrm{A}}$ is $80 a$. Since the periodic boundary condition is adopted for each side of the system, the system is symmetric for the positions $\mathrm{B}_{1}$ and $\mathrm{B}_{2}$ from the center of the system (the length of each domain is $L_{\mathrm{H}}=40 a$ ). For the simulation, the cell size is $\Delta z / a=1 / 5$ to simulate the molecular collision and the ensemble average of the macroscopic variables in Eq. (14).

To realize steady net evaporation and condensation by using the constant total molecular number in the system, the shifting method of the molecules, proposed by Meland, ${ }^{26}$ is utilized. Hereafter, we explain about the shifting method for the computational domain between the positions $\mathrm{B}_{1}$ and $B_{2}$ shown in Fig. 3. Due to net evaporation and condensation, the number of molecules that move from the left- to the right-hand sides becomes larger. If the difference in the number of molecules between the right- and the left-hand sides in half of the computational domain $L_{\mathrm{H}}$ is $J_{\text {shift }}$, then all molecules are shifted by a distance $\Delta z_{\text {shift }}$ toward the right-hand side according to $z_{i}^{\prime}=z_{i}+\Delta z_{\text {shift }}$, where $z_{i}$ is the position of $i$ th molecule. The distance $\Delta z_{\text {shift }}$ is determined by

$$
J_{\text {shift }} / 2=n\left(T_{\mathrm{L} 1}\right) U \Delta z_{\text {shift }},
$$

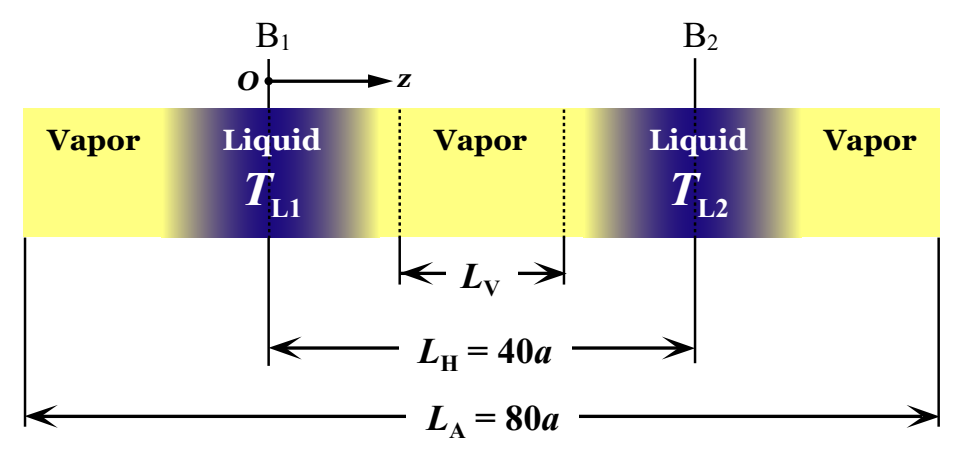

FIG. 3. Schematics of the actual simulation configuration. 
(a) Equilibrium state $T_{\mathrm{L} 1}=T_{\mathrm{L} 2}=T_{\mathrm{L}}$

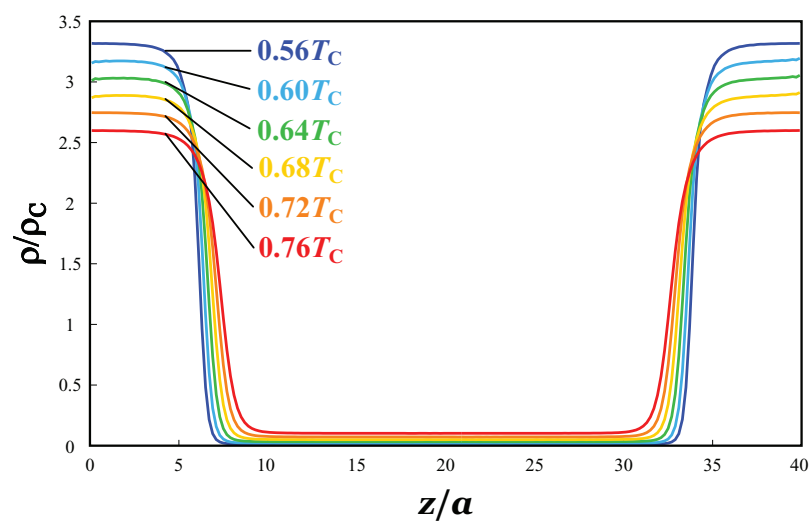

(b)

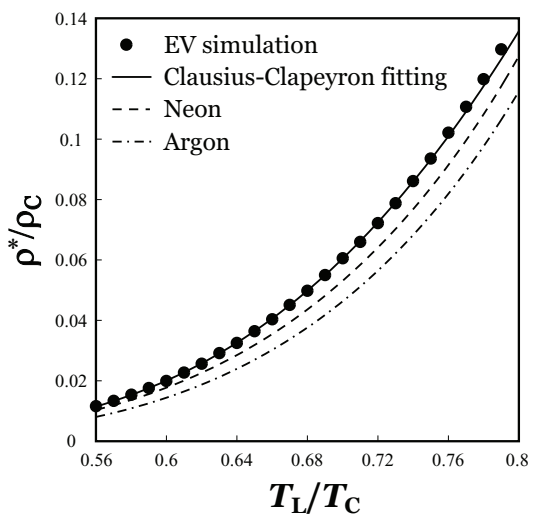

FIG. 4. Vapor-liquid equilibrium states obtained from the solutions of the Enskog-Vlasov equation: (a) density field at each temperature; (b) relationship between the saturated vapor density $\rho^{*}$ and liquid temperature $T_{\mathrm{L}}$. Each value is normalized by the critical value. The solid line is obtained from the Clausius-Clapeyron equation.

where $U$ is the unit area as the cross section of the system and $n\left(T_{\mathrm{L} 1}\right)$ is the number density of the liquid at the temperature $T_{\mathrm{L} 1}$. Molecules that are moved outside of the domain $\left(L_{\mathrm{H}}<z_{i}^{\prime}\right)$ are inserted on the left side liquid at the temperature $T_{\mathrm{L} 1}$. The position of molecule inserted on the left side liquid $z_{i}^{\prime \prime}$ is determined by $z_{i}^{\prime \prime}=z_{i}^{\prime}-L_{\mathrm{H}}$.

To maintain the constant liquid temperatures $T_{\mathrm{L} 1}$ and $T_{\mathrm{L} 2}$, the velocity scaling method is applied within the bulk liquid ${ }^{15}$ at each time step. The time increment is $\Delta t /\left(a / \sqrt{2 R T_{\mathrm{C}}}\right)=0.001$. In this simulation, we define that the bulk liquid is part of $3 a$ from the center of the density transition layer (see Fig. 4(a), the density transition layer is observed between the bulk vapor and liquid regions).

To solve the boundary value problem of the Boltzmann equation, we require the uniform net mass flux $\rho v_{z}$, the saturated vapor density $\rho^{*}$, and the Knudsen number Kn obtained from the simulations of the Enskog-Vlasov equation. $\rho v_{z}$ is adopted for the KBC in Eqs. (8) and (9), and $\rho^{*}$ and $\mathrm{Kn}$ are used to determine the length of the computational domain for the Boltzmann equation. These quantities are obtained as follows.

Various uniform net mass fluxes $\rho v_{z}$ are obtained from the simulations of the Enskog-Vlasov equation by changing the liquid temperatures $T_{\mathrm{L} 1} / T_{\mathrm{C}}$ and $T_{\mathrm{L} 2} / T_{\mathrm{C}}$. In the present paper, we focus on the KBC with the liquid temperature $T_{\mathrm{L}} / T_{\mathrm{C}}=0.60$ as the reference temperature. $T_{\mathrm{L}} / T_{\mathrm{C}}=0.60$ is a low liquid temperature. For example, $T_{\mathrm{L}} / T_{\mathrm{C}}=0.60$ is near the triple point temperature for argon molecules. ${ }^{4}$ Twenty simulations are conducted for the cases of net evaporation and condensation at the reference liquid temperature as shown in Table I: when $T_{\mathrm{L} 1} / T_{\mathrm{C}}=T_{\mathrm{L}} / T_{\mathrm{C}}=0.60, T_{\mathrm{L} 2} / T_{\mathrm{C}}$ ranges from 0.56 to 0.59 . On the other hand, when $T_{\mathrm{L} 2} / T_{\mathrm{C}}=T_{\mathrm{L}} / T_{\mathrm{C}}=0.60, T_{\mathrm{L} 1} / T_{\mathrm{C}}$ ranges from 0.61 to 0.76 .

The saturated vapor densities were estimated by performing the equilibrium simulations for the Enskog-Vlasov equation. Figure 4(a) shows the density profiles in vapor-liquid equilibrium states at

TABLE I. Simulation conditions in the present study: the liquid temperatures $T_{\mathrm{L} 1} / T_{\mathrm{C}}$ and $T_{\mathrm{L} 2} / T_{\mathrm{C}}$, the Knudsen number in the vapor phase, and the compression factor $Z$ defined as $Z=p^{*}\left(T_{0}\right) / \rho^{*}\left(T_{0}\right) R T_{0}=\left(1+\eta+\eta^{2}-\eta^{3}\right) /(1-\eta)^{3}$. If $T_{\mathrm{L} 1} / T_{\mathrm{C}}$ is the reference liquid temperature 0.60 , we denote the condition as the case of net evaporation and $T_{\mathrm{L} 2} / T_{\mathrm{C}}=0.60$ is the case of net condensation.

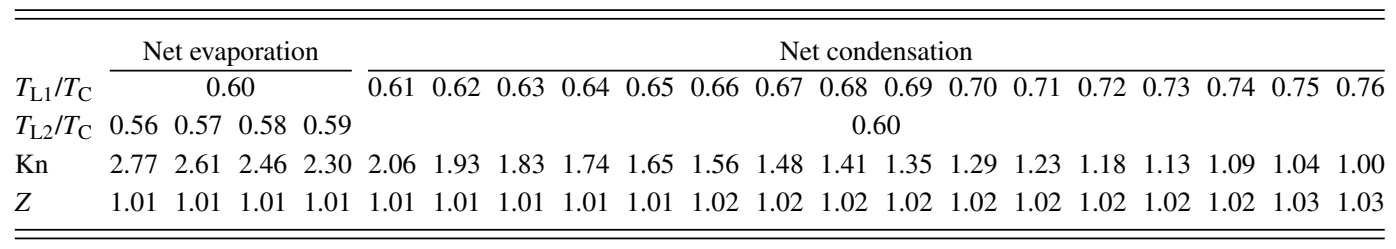


$T_{\mathrm{L} 1}=T_{\mathrm{L} 2}$ for various temperatures obtained from the simulations of the Enskog-Vlasov equation. The liquid phase is confined to the regions around the left and right edges. The temperature dependency of the density profile is the same as that for the previous simulation. ${ }^{15,23}$ The relation between the saturated vapor density and the liquid temperature can be established from these results. Figure 4(b) illustrates the relation between the saturated vapor density $\rho^{*}$ and the liquid temperature $T_{\mathrm{L}}$ obtained from the equilibrium simulation, as shown in Fig. 4(a). The relations for experimental data of argon and neon ${ }^{37}$ are also shown. As shown in the figure, the values obtained from the solutions of the Enskog-Vlasov equation are slightly different from those of argon and neon. However, these values take same tendency with the liquid temperatures in equilibrium states. The solid line is obtained from the Clausius-Clapeyron equation assuming an ideal gas

$$
\frac{\rho^{*}}{\rho_{\mathrm{C}}}=\frac{A}{R T_{\mathrm{C}}} \frac{T_{\mathrm{C}}}{T_{\mathrm{L}}} \exp \left(-\frac{\Delta H}{R T_{\mathrm{C}}} \frac{T_{\mathrm{C}}}{T_{\mathrm{L}}}\right),
$$

where the nondimensional constant $A / R T_{\mathrm{C}}$ and nondimensional latent heat $\Delta H / R T_{\mathrm{C}}$ were obtained from the nonlinear least squares method within the $T_{\mathrm{L}} / T_{\mathrm{C}}$ ranges from 0.56 to $0.70: A / R T_{\mathrm{C}}$ is 79.72 and $\Delta H / R T_{\mathrm{C}}$ is 5.279 . As can be observed, the saturated vapor density is very well fitted by the Clausius-Clapeyron equation. Similar results were reported by Frezzotti et al. ${ }^{15}$ Hence, we can evaluate the saturated vapor density from Eq. (18).

The Knudsen number $\mathrm{Kn}\left(=\ell_{0} / L_{\mathrm{V}}\right)$ is evaluated by using the length $L_{\mathrm{V}}$ of the vapor phase between kinetic boundaries at $T_{\mathrm{L} 1}$ and $T_{\mathrm{L} 2}$ and the mean free path $\ell_{0}=1 /\left(\sqrt{2} \pi a^{2} n\left(T_{0}\right) Y\left(n\left(T_{0}\right)\right)\right)$, where $T_{0}=\left(T_{\mathrm{L} 1}+T_{\mathrm{L} 2}\right) / 2$. The detailed definition of the positions of kinetic boundaries are explained in Sec. IV A. In this simulation, the range of the Knudsen number was varied from 1.00 to 2.77 as shown in Table I.

To simulate the Boltzmann equation for the above cases, the vapor must be an ideal gas. In the vapor phase, the second term in the right-hand side of Eq. (15) can be neglected. Hence, we estimated the compression factor $Z$ in the vapor phase as

$$
Z=\frac{p^{*}\left(T_{0}\right)}{\rho^{*}\left(T_{0}\right) R T_{0}}=\frac{1+\eta\left(T_{0}\right)+\eta\left(T_{0}\right)^{2}-\eta\left(T_{0}\right)^{3}}{\left(1-\eta\left(T_{0}\right)\right)^{3}},
$$

where $p^{*}$ is the saturated vapor pressure. From the results, the values of $Z$ are $1.00-1.03$ for the present simulation cases (see Table I): the present simulation is assumed as an ideal gas.

\section{B. Simulation of the Gaussian-BGK (GBGK) Boltzmann}

Once the uniform net mass flux $\rho v_{z}$ in the vapor phase is obtained from the simulation of the Enskog-Vlasov equation as explained above, the boundary condition is specified by using Eqs. (8) and (9), and we can solve the vapor flow from the Boltzmann equation. The Gaussian-BGK Boltzmann equation $^{38}$ is solved in this study to fit the Prandtl number of hard sphere molecules as $\operatorname{Pr}=0.6607^{1}$ (hereafter, we refer to this equation as the GBGK Boltzmann equation). The GBGK Boltzmann equation is written as

$$
\frac{\partial f}{\partial t}+\xi_{i} \frac{\partial f}{\partial x_{i}}=\frac{p}{\mu(1-v)}[G(f)-f],
$$

where $\mu$ is the viscosity coefficient and $v$ is the constant value used to fit the Prandtl number Pr

$$
\operatorname{Pr}=\frac{1}{1-v} .
$$

In this simulation, the value of $v$ is $1 / 2 . G(f)$ is written as

$$
G(f)=\frac{\rho}{\sqrt{\operatorname{det}\left(2 \pi \tau_{i j}\right)}} \exp \left(-\frac{1}{2}\left(\xi_{i}-v_{i}\right) \tau_{i j}^{-1}\left(\xi_{j}-v_{j}\right)\right),
$$

where $\tau_{i j}$ is defined as

$$
\tau_{i j}=(1-v) R T \delta_{i j}+v \Theta_{i j},
$$

where $\delta_{i j}$ is the Kronecker's delta and $\rho \Theta_{i j}$ is the stress tensor. 
The length of the system $L_{\mathrm{V}}$ used in the simulation of the GBGK Boltzmann equation is evaluated from the Knudsen number Kn and saturated vapor density $\rho^{*}\left(T_{0}\right)$, both obtained from the simulations of the Enskog-Vlasov equation. From the GBGK Boltzmann equation, the mean free path at the temperature $T_{0}$ is defined as $\ell_{0}=\sqrt{8} \mu\left(T_{0}\right) /\left(\rho^{*}\left(T_{0}\right) \sqrt{\pi R T_{0}}\right)$. By using $\ell_{0}$ and $\mathrm{Kn}$ obtained from the simulation of the Enskog-Vlasov equation, we determine the length of the system as $L_{\mathrm{V}}=\ell_{0} / \mathrm{Kn}$.

The finite differential method is used for the GBGK Boltzmann equation as a one-dimensional problem. As reported by $\mathrm{Chu},{ }^{39}$ we can eliminate the molecular velocity components $\xi_{x}$ and $\xi_{y}$ by multiplying the velocity distribution function $f$ by 1 or $\xi_{x}^{2}+\xi_{y}^{2}$ and integrating over the whole space of $\xi_{x}$ and $\xi_{y}$

$$
\left\{\begin{array}{l}
f_{z}\left(z, \xi_{z}, t\right)=\iint_{-\infty}^{\infty} f(z, \boldsymbol{\xi}, t) \mathrm{d} \xi_{x} \mathrm{~d} \xi_{y}, \\
h_{z}\left(z, \xi_{z}, t\right)=\iint_{-\infty}^{\infty}\left(\xi_{x}^{2}+\xi_{y}^{2}\right) f(z, \boldsymbol{\xi}, t) \mathrm{d} \xi_{x} \mathrm{~d} \xi_{y} .
\end{array}\right.
$$

The numerical simulation is performed for the distribution functions $f_{z}$ and $h_{z}$, and the time development of the macroscopic variables are calculated from the following equations:

$$
\rho=\int_{-\infty}^{\infty} f_{z} \mathrm{~d} \xi_{z}, \quad v_{z}=\frac{1}{\rho} \int_{-\infty}^{\infty} \xi_{z} f_{z} \mathrm{~d} \xi_{z}, \quad T=\frac{1}{3 \rho R}\left(\int_{-\infty}^{\infty}\left(\xi_{z}-v_{z}\right)^{2} f_{z} \mathrm{~d} \xi_{z}+\int_{-\infty}^{\infty} h_{z} \mathrm{~d} \xi_{z}\right) .
$$

500 and 2000 grids are utilized for the velocity space $\xi_{z}$ and physical space $z$ coordinates, respectively (a total of $1 \times 10^{6}$ grids). Non-uniform and uniform grids are utilized for the velocity and physical spaces, respectively: for the velocity space, the minimum size is $\Delta \xi_{z} / \sqrt{2 R T_{0}}=1.0 \times 10^{-4}$ near $\xi_{z}=0$ and the maximum size is $\Delta \xi_{z} / \sqrt{2 R T_{0}}=1.0 \times 10^{-2}$ far from $\xi_{z}=0$. Furthermore, for the physical space, the grid size is $\Delta z / \ell_{0} \cong 1.0 \times 10^{-4}$. From the simulation of the GBGK Boltzmann equation, $\sigma$ is calculated uniquely by using Eq. (5) to obtain the value of the condensation coefficient as shown in Eq. (10).

\section{RESULTS AND DISCUSSION}

\section{A. Simulation results of the Enskog-Vlasov equation}

The uniform net mass flux $\rho v_{z}$, which is required in the KBCs (Eqs. (8) and (9)), was obtained by numerically solving the Enskog-Vlasov equation in the case of steady net evaporation and condensation. Figures 5(a) and 5(b) show the density, velocity, and temperature profiles obtained by solving the Enskog-Vlasov equation at (a) liquid temperatures $T_{\mathrm{L} 1} / T_{\mathrm{C}}=0.62$ and $T_{\mathrm{L} 2} / T_{\mathrm{C}}=0.60$, and at (b) liquid temperatures $T_{\mathrm{L} 1} / T_{\mathrm{C}}=0.76$ and $T_{\mathrm{L} 2} / T_{\mathrm{C}}=0.60$. Figures $5(\mathrm{c})$ and $5(\mathrm{~d})$ show the uniform net mass flux $\rho v_{z}$ and density profiles obtained with the same conditions of Figs. 5(a) and 5(b), respectively. It should be noted that weak net evaporation and condensation occur in Figs. 5(a) and 5(c) due to the small temperature difference and strong net evaporation and condensation occur in Figs. 5(b) and 5(d) due to the large temperature difference. These figures confirmed that the temperature and density in the bulk liquids are maintained at the specified temperature and the equilibrium density, respectively. With the increase in the temperature difference of two liquid slabs, the vapor flow grows faster and the uniform net mass flux $\rho v_{z}$ becomes larger, due to the stronger net evaporation and condensation. A protrusion in the temperature profile is observed in the transition layer of the kinetic boundary at $T_{\mathrm{L} 2} / T_{\mathrm{C}}=0.60$ in net condensation, as shown in Fig. 5(b). A similar temperature profile was also observed in the previous MD simulation. ${ }^{40}$

With the use of the solution of the Enskog-Vlasov equation, the KBCs in net evaporation and condensation are adequately defined by substituting the uniform net mass flux $\rho v_{z}$ in Eqs. (8) and (9). It should be noted here that the definitions of the positions of the kinetic boundaries at which the KBCs are prescribed for solving the Boltzmann equation are ambiguous. The central positions of the density transition layers, whose 10-90 thickness is $\delta$, at the liquid temperatures $T_{\mathrm{L} 1}$ and $T_{\mathrm{L} 2}$ are $z_{\mathrm{m}}^{\mathrm{L} 1}$ and $z_{\mathrm{m}}^{\mathrm{L} 2}$, respectively. These are regarded as the reference points. The positions of the kinetic boundaries at the liquid temperatures $T_{\mathrm{L} 1}$ and $T_{\mathrm{L} 2}$ are set at $z_{\mathrm{m}}^{\mathrm{L} 1}+\zeta \delta$ and $z_{\mathrm{m}}^{\mathrm{L} 2}-\zeta \delta$, respectively, completely outside of the liquid slabs, as shown in Fig. 5. In this study, $\zeta$ was set as 2.5 on referring to the previous results obtained by MD simulations. ${ }^{5}$ We confirmed that the solution of the Boltzmann 

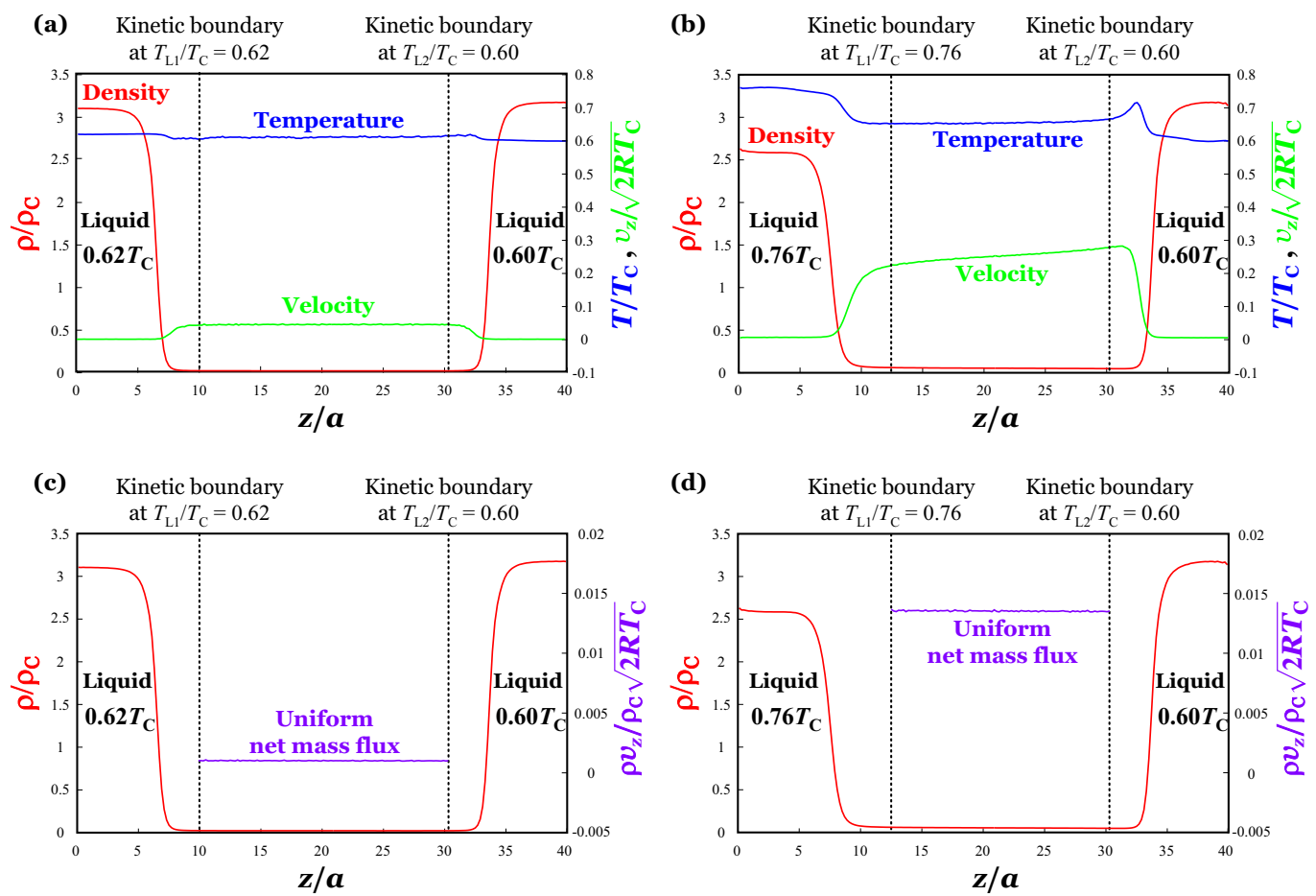

FIG. 5. Density, temperature, and velocity profiles obtained by solving the Enskog-Vlasov equation: (a) liquid temperatures $T_{\mathrm{L} 1} / T_{\mathrm{C}}=0.62$ and $T_{\mathrm{L} 2} / T_{\mathrm{C}}=0.60$; (b) liquid temperatures $T_{\mathrm{L} 1} / T_{\mathrm{C}}=0.76$ and $T_{\mathrm{L} 2} / T_{\mathrm{C}}=0.60$. (c) and (d) are the uniform net mass flux $\rho v_{z}$ in the vapor phase for (a) and (b), respectively. The all variables in the ordinate are normalized by the critical values. The abscissa is normalized by the molecular diameter $a$.

equation is rather insensitive to the positions of the kinetic boundaries, since no prominent difference was observed between the solution obtained with $\zeta=2.5$ and $\zeta=4.0$.

Figure 6 shows the normalized velocity distribution functions obtained from the solution of the Enskog-Vlasov equation at the kinetic boundaries with $\zeta=2.5$. $\hat{f}_{z}$ which is normalized as $\int_{-\infty}^{\infty} \hat{f}_{z} \mathrm{~d} \xi_{z}=1$ is the velocity distribution function normal to the kinetic boundaries. $\hat{f}_{x}$ which is normalized as $\int_{-\infty}^{\infty} \hat{f}_{x} \mathrm{~d} \xi_{x}=1$ is the velocity distribution function in the tangential direction. The solid lines are the normal and tangential components of $\hat{f}_{\text {out }}^{*}\left(T_{\mathrm{L} 1}\right)$ or $\hat{f}_{\text {out }}^{*}\left(T_{\mathrm{L} 2}\right)$, hereafter denote as $\hat{f}_{z}^{*}$ and $\hat{f}_{x}^{*}$, respectively. These distribution functions are substituted into the KBCs (Eqs. (8) and (9)) to conduct the numerical simulation of the GBGK Boltzmann equation.

Figures $6(\mathrm{a})$ and $6(\mathrm{~b})$ show the results with the cases of net condensation at the liquid temperature $T_{\mathrm{L} 2} / T_{\mathrm{C}}=0.60$. Figure 6(a) shows the case of weak net condensation $\left(T_{\mathrm{L} 1} / T_{\mathrm{C}}=0.62\right)$ and Fig. 6(b) shows the case of strong net condensation $\left(T_{\mathrm{L} 1} / T_{\mathrm{C}}=0.76\right)$. The kinetic boundary in net condensation is the right-hand side, to whom the normal direction is defined in the negative $z$ direction in this study (see Fig. 2); hence, the KBC can be prescribed if half of the velocity distribution is known, i.e., if the function is defined for $\xi_{z}<0$. In other words, in net condensation, the KBC (Eq. (9)) can be determined from the statistical information of molecules with negative velocity in this study.

In the case of weak net condensation, we can observe that the normalized velocity distribution functions $\hat{f}_{z}$ and $\hat{f}_{x}$ obtained from the solution of the Enskog-Vlasov equation are in good agreement with $\hat{f}_{z}^{*}$ and $\hat{f}_{x}^{*}$ at the liquid temperature $T_{\mathrm{L} 2} / T_{\mathrm{C}}=0.60$. In the case of strong net condensation, slight deviation of $\hat{f}_{x}$ from $\hat{f}_{x}^{*}$ is recognized: the tangential temperature $T_{\mathrm{t}} / T_{\mathrm{C}}$ estimated from $\hat{f}_{x}$ is 0.658 . It is also noted that there exists prominent deviation of $\hat{f}_{z}$ from $\hat{f}_{z}^{*}$ in vicinity of $\xi_{z} / \sqrt{2 R T_{\mathrm{L} 2}}=0$ as also reported in the recent study. ${ }^{40} \mathrm{We}$ conclude that $\hat{f}_{\mathrm{out}}^{*}\left(T_{\mathrm{L} 2}\right)$ can be properly employed as the normalized velocity distribution function $\hat{f}_{\text {out }}$ required in Eq. (9) in the case of weak net condensation.

Figure 6(c) shows the results with the case of net evaporation at the liquid temperature $T_{\mathrm{L} 1} / T_{\mathrm{C}}$ $=0.60\left(T_{\mathrm{L} 2} / T_{\mathrm{C}}=0.56\right)$. The kinetic boundary in net evaporation is the left-hand side, to whom the normal direction is defined in the positive $z$ direction in this study. Again, the KBC can be prescribed 
(a) $T_{\mathrm{L} 2} / T_{\mathrm{C}}=0.60\left(T_{\mathrm{L} 1} / T_{\mathrm{C}}=0.62\right)$
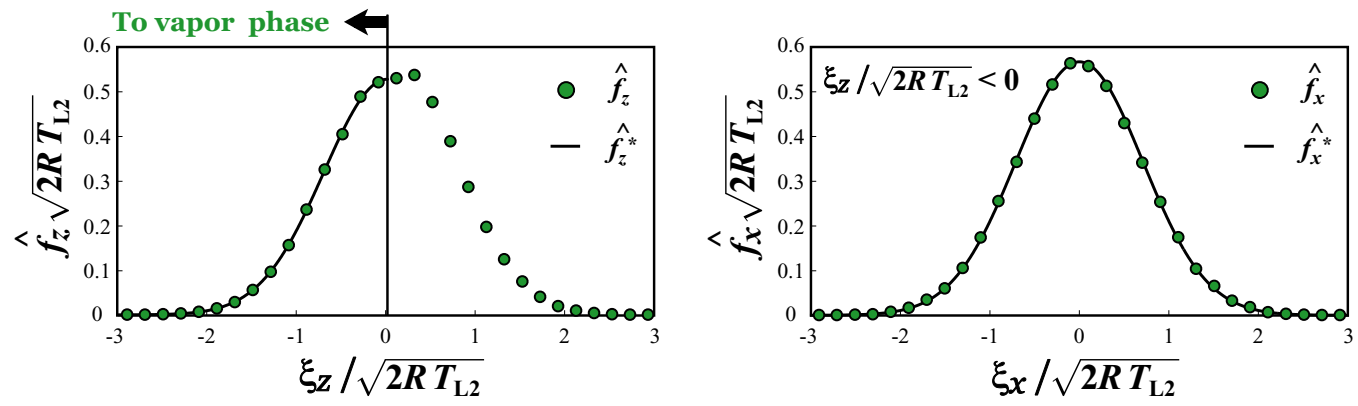

(b) $T_{\mathrm{L} 2} / T_{\mathrm{C}}=0.60\left(T_{\mathrm{L} 1} / T_{\mathrm{C}}=0.76\right)$
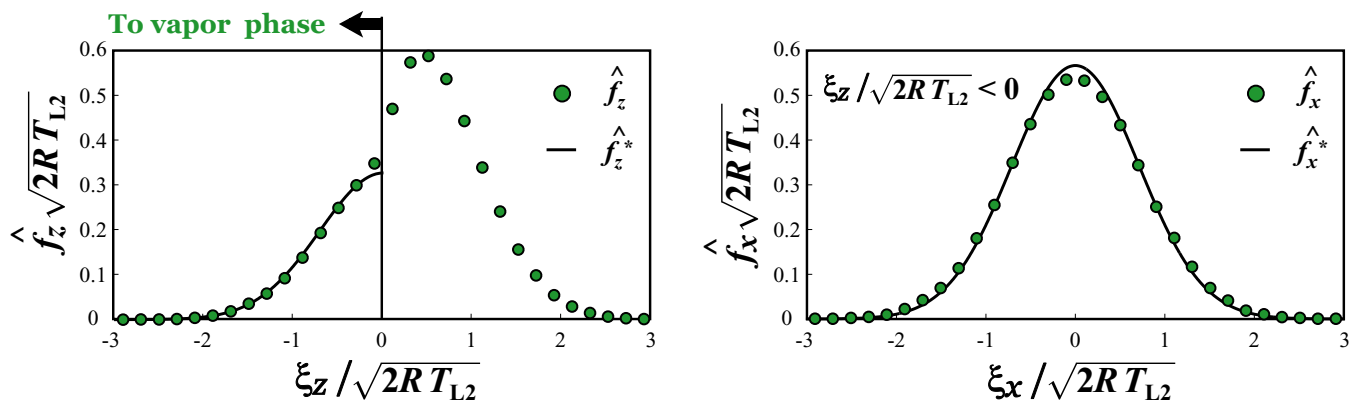

(c) $T_{\mathrm{L} 1} / T_{\mathrm{C}}=0.60\left(T_{\mathrm{L} 2} / T_{\mathrm{C}}=\mathbf{0 . 5 6}\right)$
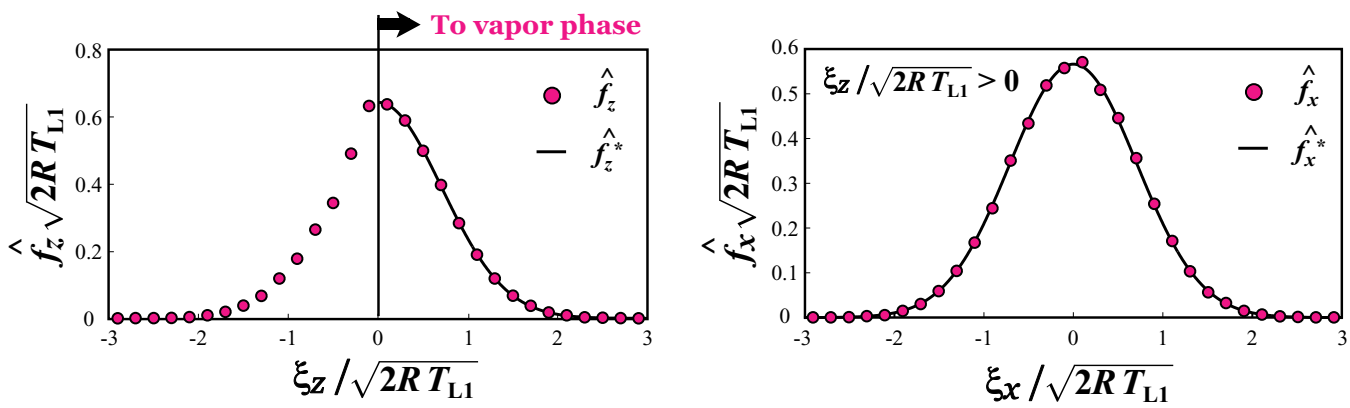

FIG. 6. Velocity distribution functions at the kinetic boundary: (a) kinetic boundary in net condensation at $T_{\mathrm{L} 2} / T_{\mathrm{C}}=0.60$ $\left(T_{\mathrm{L} 1} / T_{\mathrm{C}}=0.62\right)$; (b) kinetic boundary in net condensation at $T_{\mathrm{L} 2} / T_{\mathrm{C}}=0.60\left(T_{\mathrm{L} 1} / T_{\mathrm{C}}=0.76\right)$; (c) kinetic boundary in net evaporation at $T_{\mathrm{L} 1} / T_{\mathrm{C}}=0.60\left(T_{\mathrm{L} 2} / T_{\mathrm{C}}=0.56\right)$.

if half of the velocity distribution function is known; however, in this case, the function must be defined for $\xi_{z}>0$. In other words, in net evaporation, the KBC (Eq. (8)) can be determined from the statistical information of molecules with positive velocity in this study. We can observe that the normalized velocity distribution functions $\hat{f}_{z}$ and $\hat{f}_{x}$ obtained from the solution of the EnskogVlasov equation are in good agreement with $\hat{f}_{z}^{*}$ and $\hat{f}_{x}^{*}$ at the liquid temperature $T_{\mathrm{L} 1} / T_{\mathrm{C}}=0.60$. We also conclude that $\hat{f}_{\text {out }}^{*}\left(T_{\mathrm{L} 1}\right)$ can be properly employed as the normalized velocity distribution function $\hat{f}_{\text {out }}$ required in Eq. (8) in the case of net evaporation.

\section{B. Validation of kinetic boundary conditions}

We obtained the macroscopic variables for vapor flow field by solving the GBGK Boltzmann equation numerically with the properly defined KBCs (Eqs. (8) and (9)). These macroscopic variables are the solutions of the boundary value problem that is solely determined by the KBCs; hence, if the KBCs properly defined, the solutions obtained by the GBGK Boltzmann and Enskog-Vlasov 
(a) Density

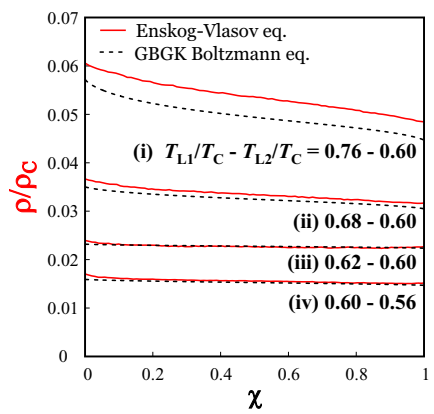

(b) Velocity

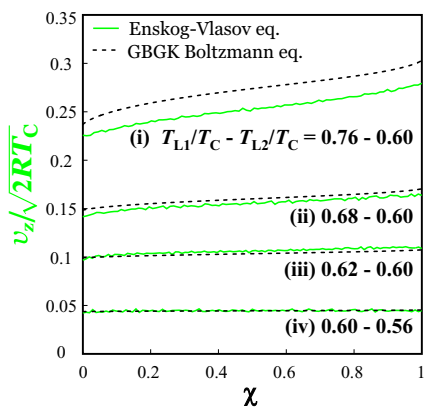

(c) Temperature

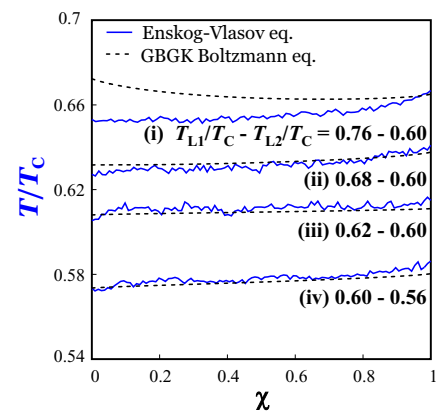

FIG. 7. Comparison between density, velocity, and temperature profiles in the vapor phase obtained by solving the EnskogVlasov and GBGK Boltzmann equations. The abscissa $\chi=0$ denotes the position of the kinetic boundary at $T_{\mathrm{L} 1}$ and $\chi=1$ that of the kinetic boundary at $T_{\mathrm{L} 2}$, respectively.

equations should exactly match. The next task is to validate the KBCs by comparing the macroscopic variables obtained from the solutions of these two equations.

Figure 7 shows the density, velocity, and temperature profiles obtained from the solutions of the Enskog-Vlasov equation and the GBGK Boltzmann equation for four typical cases, i.e., (i) $T_{\mathrm{L} 1} / T_{\mathrm{C}}$ $=0.76, T_{\mathrm{L} 2} / T_{\mathrm{C}}=0.60$, (ii) $T_{\mathrm{L} 1} / T_{\mathrm{C}}=0.68, T_{\mathrm{L} 2} / T_{\mathrm{C}}=0.60$, (iii) $T_{\mathrm{L} 1} / T_{\mathrm{C}}=0.62, T_{\mathrm{L} 2} / T_{\mathrm{C}}=0.60$, and (iv) $T_{\mathrm{L} 1} / T_{\mathrm{C}}=0.60, T_{\mathrm{L} 2} / T_{\mathrm{C}}=0.56$ : net condensation occurs at the kinetic boundary at the reference liquid temperature $T_{\mathrm{L} 2} / T_{\mathrm{C}}=0.60$ in the first three cases, and net evaporation occurs at the kinetic boundary at the reference liquid temperature $T_{\mathrm{L} 1} / T_{\mathrm{C}}=0.60$ in the last case. The abscissa $\chi$ is the normalized distance from the left to the right positions of kinetic boundaries: $\chi=0$ denotes the position of the left kinetic boundary at $T_{\mathrm{L} 1}$, and $\chi=1$ denotes that of the right kinetic boundary at $T_{\mathrm{L} 2}$.

During net evaporation (case (iv)), the macroscopic variables obtained from the GBGK Boltzmann equation are in good agreement with those obtained from the Enskog-Vlasov equation. On the other hand, during net condensation (cases (i)-(iii)), the deviation of the macroscopic variables obtained from the GBGK Boltzmann equation from those obtained from the Enskog-Vlasov equation becomes larger as the net condensation strengthens, that is, as $T_{\mathrm{L} 1} / T_{\mathrm{C}}$ increases. It should be noted that the inverted temperature gradient profile disappears in the solution of the GBGK Boltzmann equation in the case (i). This result reveals that the KBCs are not properly defined in the case (i) since the assumption of $\hat{f}_{\text {out }}^{*}\left(T_{\mathrm{L}}\right)$ is employed as the normalized velocity distribution functions in Eqs. (8) and (9). We conclude that $\hat{f}_{\text {out }}^{*}\left(T_{\mathrm{L}}\right)$ is appropriate to be employed as the normalized velocity distribution function of the KBCs when the cases of net evaporation and weak net condensation; the maximum deviation of the macroscopic variables obtained from the GBGK Boltzmann equation from those obtained from the Enskog-Vlasov equation is less than 5\%. It should be noted that the result with $T_{\mathrm{L} 1} / T_{\mathrm{C}}=0.68$ (ii) is the criteria case with the maximum deviation of less than $5 \%$ in the present study.

Hereafter, we consider the kinetic boundary in net evaporation or condensation at the reference liquid temperature as $T_{\mathrm{L}} / T_{\mathrm{C}}=0.60$. For the convenience of the following discussion, we regard $J_{\text {coll }} / J_{\text {coll }}^{*}=\sigma / \rho^{*}$ as the index of the degree of nonequilibrium, where $J_{\text {coll }}^{*}$ is the collision molecular mass flux in equilibrium state defined as $J_{\text {coll }}^{*}=J_{\text {out }}^{*}=\rho^{*} \sqrt{R T_{\mathrm{L}} / 2 \pi}$. When the vapor-liquid equilibrium state, $\sigma / \rho^{*}$ equals unity. On the other hand, at the kinetic boundaries in net evaporation and condensation, the values become $\sigma / \rho^{*}<1$ and $\sigma / \rho^{*}>1$, respectively. On the basis of the above discussion, the degree of nonequilibrium $\sigma / \rho^{*}$, for which the normalized velocity distribution function $\hat{f}_{\text {out }}$ can be assumed as $\hat{f}_{\text {out }}^{*}\left(T_{\mathrm{L}}\right)$, ranges from $0.6<\sigma / \rho^{*}<2.3$.

\section{Determination of the evaporation/condensation coefficients and construction of kinetic boundary conditions}

As discussed in Sec. II, various mass fluxes are defined at the kinetic boundary as shown in Fig. 2. In order to determine the evaporation and condensation coefficients, we investigate the mutual 
(a) Kinetic boundary in net evaporation
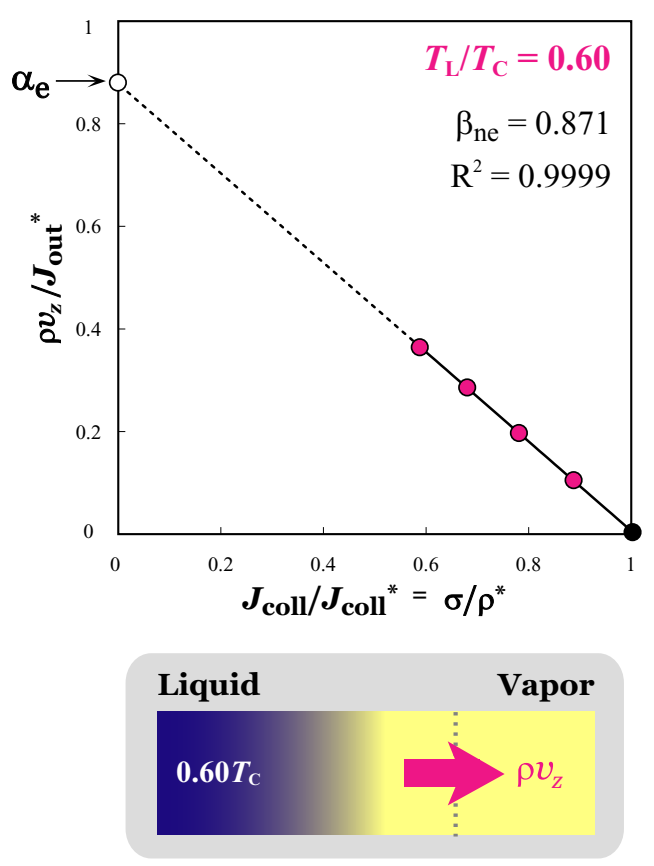

(b) Kinetic boundary in net condensation
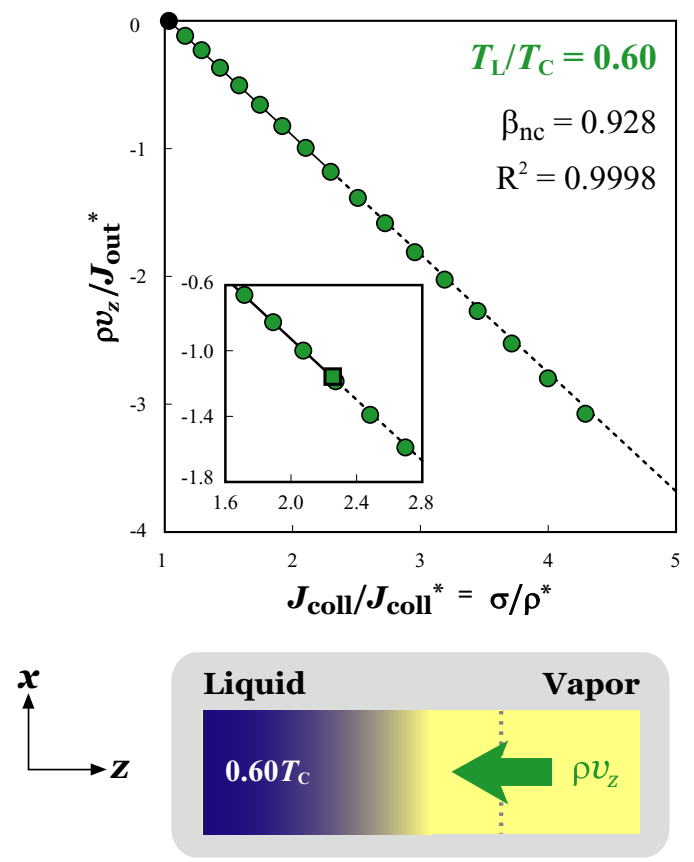

FIG. 8. Relationships between the uniform net mass flux and $\sigma / \rho^{*}$ at the kinetic boundaries: (a) kinetic boundary in net evaporation at $T_{\mathrm{L}} / T_{\mathrm{C}}=0.60$; (b) kinetic boundary in net condensation at $T_{\mathrm{L}} / T_{\mathrm{C}}=0.60 . J_{\text {coll }}^{*}=J_{\text {out }}^{*}=\rho^{*} \sqrt{R T_{\mathrm{L}} / 2 \pi}$.

relationships between these fluxes by further analysis of the solutions of the Enskog-Vlasov and GBGK Boltzmann equations. We evaluate these coefficients from 20 cases for various temperature differences between left- and right-hand liquid slabs. The relation between the uniform net mass flux $\rho v_{z}$ normalized by the outgoing molecular mass flux at an equilibrium state $J_{\text {out }}^{*}$, and the degree of nonequilibrium $\sigma / \rho^{*}$ at the kinetic boundary at the reference liquid temperature $T_{\mathrm{L}} / T_{\mathrm{C}}=0.60$ is shown in Fig. 8. Figures 8(a) and 8(b) show the cases of net evaporation and condensation, respectively. As already explained, we obtained the uniform net mass flux $\rho v_{z}$ from the solution of the Enskog-Vlasov equation and $\sigma$ from the solution of the GBGK Boltzmann equation with the use of the obtained $\rho v_{z}$. In the following discussion, notably, the net evaporation occurs at the kinetic boundary if the uniform net mass flux $\rho v_{z}>0$; conversely, net condensation occurs if $\rho v_{z}<0$ (see bottom schematics in Fig. 8). When $T_{\mathrm{L} 1}$ equals $T_{\mathrm{L} 2}$, the degree of nonequilibrium is $\sigma / \rho^{*}=1$ and the uniform net mass flux is $\rho v_{z}=0$, i.e., the flow field in the vicinity of each kinetic boundary is in an equilibrium state. With the increase in the temperature difference between $T_{\mathrm{L} 1}$ and $T_{\mathrm{L} 2}$, the deviation of $\sigma / \rho^{*}$ from unity increases, i.e., the degree of nonequilibrium increases.

It is the most striking finding that the uniform net mass flux $\rho v_{z}$ is well described as a linear function of the degree of nonequilibrium $\sigma / \rho^{*}$. We employed linear regression analyses with the use of the following function when $0.6<\sigma / \rho^{*} \leq 1$ and $1 \leq \sigma / \rho^{*}<2.3$, for the cases of the net evaporation and condensation, respectively:

$$
\begin{array}{ll}
\frac{\rho v_{z}}{J_{\text {out }}^{*}}=\beta_{\mathrm{ne}}\left(1-\frac{\sigma}{\rho^{*}}\right), & \text { when } 0.6<\frac{\sigma}{\rho^{*}} \leq 1, \\
\frac{\rho v_{z}}{J_{\text {out }}^{*}}=\beta_{\mathrm{nc}}\left(1-\frac{\sigma}{\rho^{*}}\right), & \text { when } 1 \leq \frac{\sigma}{\rho^{*}}<2.3,
\end{array}
$$

where $\beta_{\mathrm{ne}}$ and $\beta_{\mathrm{nc}}$ are regression coefficients, calculated as 0.871 and 0.928 , respectively. The obtained fittings completely match the data, where coefficients of determination, $R^{2}$ for the cases of net evaporation and condensation are 0.9999 and 0.9998 , respectively. One of the authors previously conducted experiments on water and methanol, and has already proposed a linear relationship 
between net mass flux and the degree of nonequilibrium, ${ }^{25}$ however, the $R^{2}$ values of water and methanol were 0.51 and 0.91 , respectively. In the present study, we can clearly show that the uniform net mass flux $\rho v_{z}$ is well described as linear function of the degree of nonequilibrium $\sigma / \rho^{*}$, owing to the small statistical errors from the solution of the Enskog-Vlasov equation.

We also confirmed that the results are totally insensitive to the length of the vapor phase, $L_{\mathrm{V}}$, as shown in Fig. 3. The uniform net mass flux $\rho v_{z}$ was obtained by the solution of the Enskog-Vlasov equation with the conditions where $L_{\mathrm{A}}=160 a, \mathrm{Kn}=0.462, T_{\mathrm{L} 1} / T_{\mathrm{C}}=0.68$, and $T_{\mathrm{L} 2} / T_{\mathrm{C}}=0.60$. The result is shown as the green square in the enlarged view in Fig. 8(b). We conclude that the uniform net mass flux $\rho v_{z}$ at the specified degree of nonequilibrium $\sigma / \rho^{*}$ is independent of $L_{\mathrm{V}}$.

When $\sigma / \rho^{*}=0$ in Eq. (26), the only flux created at the kinetic boundary is the evaporation molecular mass flux $J_{\text {evap }}$ since no collision mass flux $J_{\text {coll }}$ exists: hence, from Eqs. (6) and (7), $J_{\text {evap }}$ becomes identical to $\rho v_{z}$, which leads to the conclusion that $\beta_{\text {ne }}$ in Eq. (26) is regarded as the evaporation coefficient $\alpha_{\mathrm{e}}$. The difference between the above obtained value of $\beta_{\text {ne }}$ that is 0.871 and the one that is 0.880 obtained from the solution of the Enskog-Vlasov equation with the use of the vacuum evaporation simulation ${ }^{4}$ is about $1 \%$; hence, we propose that we invented the alternative evaluation of the evaporation coefficient. In the present study, the value of $\alpha_{\mathrm{e}}$ is set to be equal to that of $\beta_{\text {ne }}, 0.871$. Figure 9(a) shows the evaporation coefficients obtained from the present study and recent MD studies. The circle denotes the result of the present study, the square and triangle denote the results for argon, ${ }^{16,22}$ and solid curve is obtained from MD simulations for argon, water, and methanol with the virtual vacuum simulation. ${ }^{6}$ From these results, we cannot see a noticeable difference between our simulation and the MD results ${ }^{6,22}$ at $T_{\mathrm{L}} / T_{\mathrm{C}}=0.60$. Substituting the above defined $\alpha_{\mathrm{e}}$ into Eq. (10), we obtained the condensation coefficient $\alpha_{\mathrm{c}}$. Figure 9(b) shows the relationship between the condensation coefficient $\alpha_{\mathrm{c}}$ and the degree of nonequilibrium $\sigma / \rho^{*}$. During net condensation, the condensation coefficient increases with the increase in the degree of nonequilibrium, while during net evaporation, it is independent of the degree of nonequilibrium.

With the use of the linear relation as shown in Fig. 8, that is, by substituting Eq. (26) into Eq. (10), we obtained the explicit form of the condensation coefficient for the case of net evaporation

$$
\alpha_{\mathrm{c}}=\alpha_{\mathrm{e}}=\beta_{\mathrm{ne}}, \quad \text { when } 0.6<\frac{\sigma}{\rho^{*}} \leq 1,
$$

where the relation $\beta_{\mathrm{ne}}=\alpha_{\mathrm{e}}$, as explained in above discussion, is used. For the case of net condensation, we can also obtain the following equation by substituting Eq. (27) into Eq. (10):

$$
\alpha_{\mathrm{c}}=\frac{\rho^{*}}{\sigma}\left(\alpha_{\mathrm{e}}-\beta_{\mathrm{nc}}\right)+\beta_{\mathrm{nc}}, \quad \text { when } 1 \leq \frac{\sigma}{\rho^{*}}<2.3 .
$$

The line plots shown in Fig. 9(a) predicted by Eqs. (28) and (29).

(a)

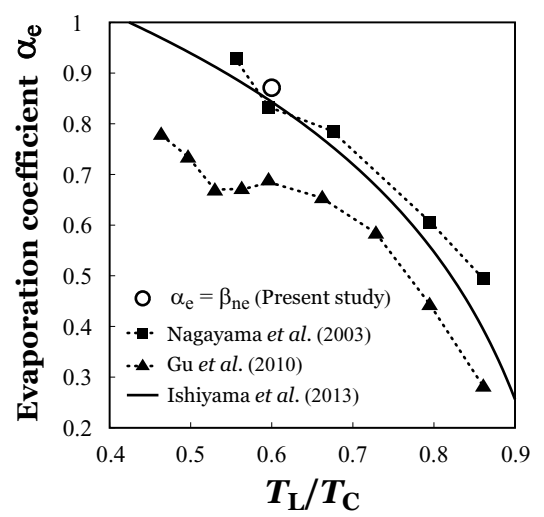

(b)

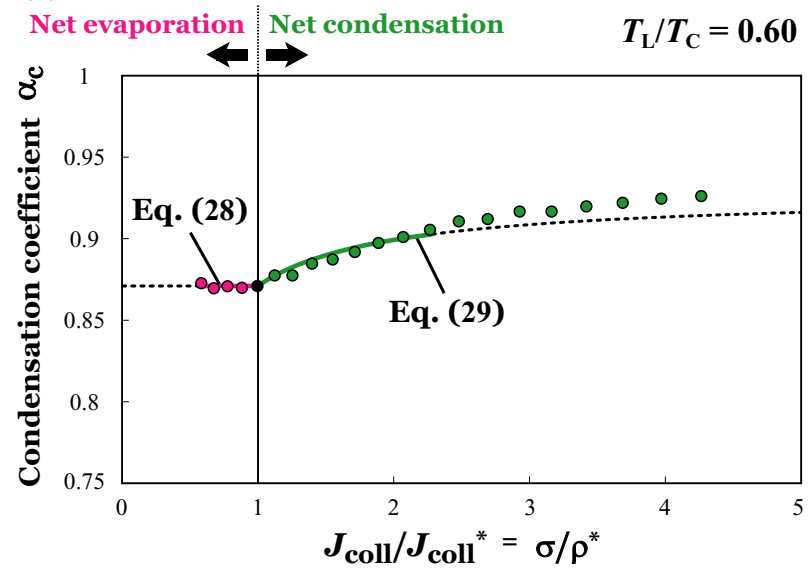

FIG. 9. (a) Comparison of evaporation coefficients; (b) condensation coefficient versus $\sigma / \rho^{*}$ at the reference liquid temperature $T_{\mathrm{L}} / T_{\mathrm{C}}=0.60$. 
It is important to emphasize that the proposed method to determine the $\mathrm{KBC}$ of the Boltzmann equation enables us to evaluate both the accuracy of the results by considering the error in macroscopic variables that depend on the degree of nonequilibrium and the values of condensation and evaporation coefficients that also depend on the degree of nonequilibrium. Needless to say that quantitative evaluation of these coefficients depends on the model describing the molecular interaction; however, the method itself proposed is universally applicable regardless to the model.

The uniform net mass flux $\rho v_{z}$ can be obtained either by solving the Enskog-Vlasov equation (for a Sutherland potential) or by conducting MD simulation (if adopting a more practical potential such as the Lennard-Jones potential). In both cases, the evaporation and condensation coefficients can be obtained by solving the Boltzmann equation with $\rho v_{z}$ substituting into Eqs. (8) and (9). Moreover, the hard sphere molecules in the present system are replaced with polyatomic molecules and the uniform net mass flux $\rho v_{z}$ in the vapor phase is obtained by MD simulations, the boundary value problem with KBCs, given by Eqs. (8) and (9), can be solved using the polyatomic version of the GBGK Boltzmann equation. ${ }^{38}$ Numerical simulations of the GBGK Boltzmann equation for polyatomic molecules are presented in Fujikawa et $_{\text {al. }}{ }^{3}$ and Kobayashi et al..$^{25}$

Furthermore, we can propose the new KBCs to describe the vapor flow accompanied with net evaporation and condensation from the above results. By substituting Eq. (28) or (29) into Eq. (2), we can obtain the KBCs in net evaporation and condensation without using the condensation and evaporation coefficients

$$
\begin{aligned}
& f_{\text {out }}=\frac{\left[\beta_{\mathrm{ne}} \rho^{*}+\left(1-\beta_{\mathrm{ne}}\right) \sigma\right]}{\left(\sqrt{2 \pi R T_{\mathrm{L}}}\right)^{3}} \exp \left(-\frac{\xi_{x}^{2}+\xi_{y}^{2}+\xi_{z}^{2}}{2 R T_{\mathrm{L}}}\right), \quad \text { for } \xi_{z}>0, \quad \text { when } 0.6<\frac{\sigma}{\rho^{*}} \leq 1, \\
& f_{\text {out }}=\frac{\left[\beta_{\mathrm{nc}} \rho^{*}+\left(1-\beta_{\mathrm{nc}}\right) \sigma\right]}{\left(\sqrt{2 \pi R T_{\mathrm{L}}}\right)^{3}} \exp \left(-\frac{\xi_{x}^{2}+\xi_{y}^{2}+\xi_{z}^{2}}{2 R T_{\mathrm{L}}}\right), \quad \text { for } \xi_{z}>0, \quad \text { when } 1 \leq \frac{\sigma}{\rho^{*}}<2.3,
\end{aligned}
$$

where $T_{\mathrm{L}} / T_{\mathrm{c}}$ is set to 0.60 in the present study. The above equations are similar to the conventional KBC that is widely used in the kinetic theory. ${ }^{1,2}$ The difference between $\beta_{\text {ne }}$ and $\beta_{\mathrm{nc}}$, and the detailed dynamics of molecular motion at the kinetic boundary are not clear in the present study. However, by using constant parameters $\beta_{\text {ne }}$ and $\beta_{\text {nc }}$, we can analyze the correct vapor flows. It is one of the important results of this study that we do not have to change the value of condensation coefficient according to the value of $\sigma / \rho^{*}$ as shown in Fig. 9, i.e., we can specify the KBC by simply knowing whether $\sigma / \rho^{*}$ is larger or smaller than unity.

\section{CONCLUSIONS}

In the present study, the $\mathrm{KBCs}$ in net evaporation and condensation were investigated by combining the simulations of the Enskog-Vlasov and Gaussian-BGK Boltzmann equations for the two-surface problem. We proposed a state-of-the-art method for determining the evaporation and condensation coefficients from the uniform net mass flux that accompanies net evaporation and condensation. The proposed novel method can validate the KBCs by comparing macroscopic variables of vapor phase obtained from the solutions of the Enskog-Vlasov and GBGK Boltzmann equations. Furthermore, we confirmed the velocity distribution function of the KBC. Specifically, in the present configuration, the isotropic Gaussian at the liquid temperature $T_{\mathrm{L}} / T_{\mathrm{C}}=0.60 \mathrm{can}$ be assumed as the velocity distribution function of the $\mathrm{KBC}$ in $0.6<\sigma / \rho^{*}<2.3$.

From the simulation results, we evaluated the value of the evaporation coefficient without the vacuum evaporation simulation. Then, the condensation coefficient was determined by using the evaporation coefficient. When the vapor-liquid system is in net evaporation, the condensation and evaporation coefficients are identical and constant. On the other hand, when the vapor-liquid system is in net condensation, the value of the condensation coefficient increases with the degree of nonequilibrium $\sigma / \rho^{*}$. Furthermore, the kinetic boundary condition was proposed with the constant parameters ( $\beta_{\mathrm{ne}}$ and $\left.\beta_{\mathrm{nc}}\right)$ instead of the evaporation and condensation coefficients in net evaporation and condensation. However, the present result for the condensation coefficient shows an inverse 
tendency to those obtained from the previous experiment in net condensation. ${ }^{25}$ The discrepancy of the values between the molecular simulations and experiments remains an open question. The influences of the internal structure and binary component of the molecules for the $\mathrm{KBC}$ in net evaporation or condensation are also unsolved problems.

\section{ACKNOWLEDGMENTS}

This study was partly supported by JSPS Grant-in-Aid for Young Scientists (B) (25820038).

${ }^{1}$ Y. Sone, Molecular Gas Dynamics: Theory, Techniques and Applications (Birkhäuser, Boston, 2007).

${ }^{2}$ C. Cercignani, Rarefied Gas Dynamics: From Basic Concepts to Actual Calculations (Cambridge University Press, New York, 2000).

${ }^{3}$ S. Fujikawa, T. Yano, and M. Watanabe, Vapor-Liquid Interface, Bubble and Droplets: Fundamentals and Applications (Springer-Verlag, New York, 2011).

${ }^{4}$ T. Ishiyama, T. Yano, and S. Fujikawa, "Molecular dynamics study of kinetic boundary condition at an interface between argon vapor and its condensed phase," Phys. Fluids 16, 2899 (2004).

${ }^{5}$ T. Ishiyama, T. Yano, and S. Fujikawa, "Kinetic boundary condition at a vapor-liquid interface," Phys. Rev. Lett. 95, 084504 (2005)

${ }^{6}$ T. Ishiyama, S. Fujikawa, T. Kurz, and W. Lauterborn, "Nonequilibrium kinetic boundary condition at the vapor-liquid interface of argon,” Phys. Rev. E 88, 042406 (2013).

${ }^{7}$ M. Matsumoto and Y. Kataoka, "Dynamic processes at a liquid surface of methanol," Phys. Rev. Lett. 69, 3782 (1992).

${ }^{8}$ V. V. Zhakhovskii and S. I. Anisimov, "Molecular-dynamics simulation of evaporation of a liquid,” J. Exp. Theor. Phys. 84, 734 (1997).

${ }^{9}$ S. I. Anisimov, D. O. Dunikov, V. V. Zhakhovskii, and S. P. Malyshenko, "Properties of a liquid-gas interface at high-rate evaporation," J. Chem. Phys. 110, 8722 (1999).

${ }^{10}$ T. Tsuruta, H. Tanaka, and T. Masuoka, "Condensation/evaporation coefficient and velocity distributions at liquid-vapor interface," Int. J. Heat Mass Transfer 42, 4107 (1999).

${ }^{11}$ G. Nagayama and T. Tsuruta, "A general expression for the condensation coefficient based on transition state theory and molecular dynamics simulation,” J. Chem. Phys. 118, 1392 (2003).

${ }^{12}$ A. Frezzotti, P. Grosfils, and S. Toxvaerd, "Evidence of an inverted temperature gradient during evaporation/condensation of a Lennard-Jones fluid,” Phys. Fluids 15, 2837 (2003).

${ }^{13}$ R. Meland, A. Frezzotti, T. Ytrehus, and B. Hafskjold, "Nonequilibrium molecular-dynamics simulation of net evaporation and net condensation, and evaluation of the gas-kinetic boundary condition at the interphase," Phys. Fluids 16, 223 (2004).

${ }^{14}$ T. Tsuruta and G. Nagayama, "Molecular dynamics studies on the condensation coefficient of water," J. Phys. Chem. B 108, 1736 (2004).

${ }^{15}$ A. Frezzotti, L. Gibelli, and S. Lorenzani, "Mean field kinetic theory description of evaporation of a fluid into vacuum," Phys. Fluids 17, 012102 (2005).

${ }^{16}$ K. Gu, C. B. Watkins, and J. Koplik, "Molecular dynamics simulation of the equilibrium liquid-vapor interphase with solidification," Fluid Phase Equilib. 297, 77 (2010).

${ }^{17}$ K. Gu, C. B. Watkins, and J. Koplik, "Multiscale molecular simulations of argon vapor condensation onto a cooled substrate with bulk flow," Phys. Fluids 22, 112002 (2010).

${ }^{18}$ A. Frezzotti, "Boundary conditions at the vapor-liquid interface," Phys. Fluids 23, 030609 (2011).

${ }^{19}$ J.-F. Xie, S. S. Sazhin, and B. Y. Cao, "Molecular dynamics study of the processes in the vicinity of the n-dodecane vapour/liquid interface," Phys. Fluids 23, 112104 (2011).

${ }^{20}$ B. Y. Cao, J.-F. Xie, and S. S. Sazhin, "Molecular dynamics study on evaporation and condensation of n-dodecane at liquid-vapor phase equilibria," J. Chem. Phys. 134, 164309 (2011).

${ }^{21}$ S. Cheng, J. B. Lechman, S. J. Plimpton, and G. S. Grest, "Evaporation of Lennard-Jones fluids," J. Chem. Phys. 134, 224704 (2011).

${ }^{22}$ T. Tokunaga, G. Nagayama, and T. Tsuruta, "Non-equilibrium transport phenomena on liquid surface and energy accommodation coefficients on reflecting molecules,” Trans. Jpn. Soc. Mech. Eng. B 77, 324 (2011) (in Japanese).

${ }^{23}$ K. Kobayashi, K. Ohashi, and M. Watanabe, "Numerical analysis of vapor-liquid two-phase system based on the EnskogVlasov equation," AIP Conf. Proc. 1501, 1145 (2012).

${ }^{24}$ P. Louden, R. Schoenborn, and C. P. Lawrence, "Molecular dynamics simulations of the condensation coefficient of water," Fluid Phase Equilib. 349, 83 (2013).

${ }^{25}$ K. Kobayashi, S. Watanabe, D. Yamano, T. Yano, and S. Fujikawa, "Condensation coefficient of water in a weak condensation state," Fluid Dyn. Res. 40, 585 (2008).

${ }^{26}$ R. Meland, "Molecular dynamics simulation of the inverted temperature gradient phenomenon," Phys. Fluids $\mathbf{1 5}, 3244$ (2003).

${ }^{27}$ T. Yano, "Half-space problem for gas flows with evaporation or condensation on a planar interface with a general boundary condition," Fluid Dyn. Res. 40, 474 (2008).

${ }^{28}$ Y. Pao, "Application of kinetic theory to the problem of evaporation and condensation," Phys. Fluids 14, 306 (1971).

${ }^{29}$ Y. Onishi, "On the negative mass flows in evaporation and condensation problems," Phys. Fluids 17, 127106 (2005).

${ }^{30}$ J. J. Wleklinski, "Verification of the inverted temperature gradient condition via molecular dynamics simulation," Physica A 306, 151 (2006).

${ }^{31}$ R. Meland and T. Ytrehus, "Dependence of the inverted temperature gradient phenomenon on the condensation coefficient," Phys. Fluids 16, 836 (2004). 
${ }^{32}$ J. Karkheck and G. Stell, “Kinetic mean-field theories,” J. Chem. Phys. 75, 1475 (1981).

${ }^{33}$ N. F. Carnahan and K. E. Starling, "Equation of state for nonattracting rigid spheres," J. Chem. Phys. 51, 635 (1969).

${ }^{34}$ A. Frezzotti, "A particle scheme for the numerical solution of the Enskog equation," Phys. Fluids 9, 1329 (1997).

35 J. M. Montanero and A. Santos, "Monte Carlo simulation method for the Enskog equation," Phys. Rev. E 54, 438 (1996).

${ }^{36}$ A. Frezzotti and M. Rossi, "Slip effects at the vapor-liquid boundary," AIP Conf. Proc. 1501, 903 (2012).

${ }^{37}$ JSME Data Book: Thermophysical Properties of Fluids, edited by Japan Society of Mechanical Engineering (JSME, Tokyo, 1983) (in Japanese).

${ }^{38}$ P. Andries, P. Le Tallec, J.-P. Perlat, and B. Perthame, "The Gaussian-BGK model of Boltzmann equation with small Prandtl number," Eur. J. Mech. B/Fluids 19, 813 (2000).

${ }^{39}$ C. K. Chu, "Kinetic-theoretic description of shock wave formation. II," Phys. Fluids 8, 1450 (1965).

${ }^{40} \mathrm{~T}$. Yano, "Molecular dynamics study of nonequilibrium processes of evaporation and condensation at a vapor-liquid interface," AIP Conf. Proc. 1501, 926 (2012). 